\title{
Listeria monocytogenes
}

National Cancer Institute

\section{Source}

National Cancer Institute. Listeria monocytogenes. NCI Thesaurus. Code C86502.

A species of facultatively anaerobic, Gram positive, rod shaped bacteria in the phylum Firmicutes. This species is motile by up to four peritrichous flagella, catalase positive, hemolytic, produces acid from glucose, trehalose, levulose and salacin, indole negative and does not reduce nitrate. L. monocytogenes is a food borne pathogen, being the causative agent of listeriosis in humans and animals. 